\title{
BMJ Open Exploring stakeholder views on intervening in hospital around childhood secondhand smoke exposure (Precedent): a protocol for a qualitative study
}

\author{
Erica Ferris (D) , ${ }^{1}$ Carole Cummins, ${ }^{1}$ Christopher Chiswell, ${ }^{2}$ Laura Jones (i) ${ }^{1}$
}

To cite: Ferris E,

Cummins C, Chiswell C, et al. Exploring stakeholder views on intervening in hospital around childhood secondhand smoke exposure (Precedent): a protocol for a qualitative study. BMJ Open 2021;11:e047817. doi:10.1136/ bmjopen-2020-047817

- Prepublication history and additional supplemental material for this paper are available online. To view these files, please visit the journal online (http://dx.doi.org/10.1136/ bmjopen-2020-047817).

Received 13 December 2020 Accepted 30 September 2021

Check for updates

(C) Author(s) (or their employer(s)) 2021. Re-use permitted under CC BY-NC. No commercial re-use. See rights and permissions. Published by BMJ.

${ }^{1}$ Institute of Applied Health Research, University of Birmingham, Birmingham, UK ${ }^{2}$ Public Health, Birmingham Women's and Children's NHS Foundation Trust, Birmingham, UK

Correspondence to

Dr Laura Jones;

L.L.Jones@bham.ac.uk

\section{ABSTRACT}

Introduction Secondhand smoke exposure (SHSe) in childhood is linked with increased morbidity and mortality. Hospital or secondary care contact may present a 'teachable moment' to provide parents with support to change their home smoking behaviours to reduce children's SHSe. There is a lack of robust qualitative evidence around parents and healthcare professionals (HCPs) views on using this teachable moment to successfully initiate behavioural change. We aim to identify and understand what is important to stakeholders with a view to informing the development of a support package to help parents change their home smoking behaviours. Methods and analysis This qualitative study will be theoretically underpinned by the Capability, Opportunity and Motivation Behaviour (COM-B) model of behavioural change. It will involve semistructured interviews and/ or discussion groups with up to 20 parents who smoke and up to $25 \mathrm{HCPs}$. Stakeholders will be recruited from a single National Health Service children's hospital in England. Interviews and/or discussion groups will be audio recorded, transcribed and anonymised. The transcripts and any field notes will be analysed using the framework method. Initially, we will apply COM-B to the data deductively and will then code inductively within each domain.

Ethics and dissemination The protocol for this study received a favourable outcome from the East Midlands Leicester Central Research Ethics Committee (19/ EM/0171). Results will be written up as part of a PhD thesis, submitted for publication in peer-reviewed journals and presentation at conferences.

Trial registration number ISRCTN40084089.

\section{BACKGROUND}

Secondhand smoke exposure (SHSe) is the combination of side stream smoke (which is the smoke given off by the burning tobacco product) and mainstream smoke (which is the smoke exhaled by the smoker). ${ }^{1}$ There is no safe level of exposure. ${ }^{2}$ Globally, 1.2 million deaths are attributed to exposure to SHS each year, ${ }^{3}$ with $40 \%$ of children and young people
Strengths and limitations of this study

- The use of qualitative methods will enrich our understanding of parental receptiveness for receiving support to reduce secondhand smoke exposure (SHSe) and healthcare professionals' willingness to intervene around childhood SHSe within an English National Health Service setting.

- Theoretically underpinned by the Capability, Opportunity and Motivation Behaviour model of behavioural change.

- Recruitment and eligibility is from a single children's hospital within the National Health Service, and so findings may not be transferable to other services.

aged under 14 years regularly exposed. ${ }^{1}$ It is estimated that a third of premature deaths attributable to exposure to SHS occur in children with 65000 children each year dying from illnesses attributable to SHSe. ${ }^{3}$ While exposure to SHS in England has declined in recent years, ${ }^{4} 39 \%$ of children who live with smokers are still regularly exposed at home. ${ }^{5}$

Exposure to SHS has been causally linked with a number of childhood morbidities including respiratory tract infections, ${ }^{6}$ middle ear disease ${ }^{7}$ and meningitis. ${ }^{8}$ Children exposed to SHS are also at an increased risk of anaesthetic-related harms when undergoing planned and emergency surgical procedures. ${ }^{9}$ SHSe by parents may also be associated with the development of some childhood cancers $^{10}$ and children who are regularly exposed to SHS are three times as likely to develop lung cancer as adults, compared with children who grew up in smoke-free environments. ${ }^{11}$ In addition, children who grow up with smokers are over twice as likely to become smokers themselves ${ }^{12}$ and becoming a regular smoker doubles their risk of premature death, ${ }^{13}$ particularly from cancer. 
Childhood illnesses as a result of SHSe were estimated in 2006 to cost the National Health Service (NHS) $£ 23.3$ million annually across primary and secondary care. ${ }^{2}$ Published in 2010, the annual secondary care costs for additional hospital admissions as a result of childhood SHSe in the home are estimated to be $£ 13.6$ million. ${ }^{13}$ More broadly, the cost to the NHS for the uptake of smoking after childhood household SHSe exposure is an estimated $£ 5.7$ million in additional treatment costs. ${ }^{13}$

Parental smoking and household smoking bans are the two main determinants of a child's level of SHSe in high-income countries with comprehensive smoke-free legislation, such as the UK. ${ }^{414}$ The most effective way to reduce children's SHSe is to support their parents to quit smoking. ${ }^{15}$ However, for parents who cannot or are unable to quit, there is a need to support families to change their smoking behaviours, such as to support them to make their homes smoke-free to reduce the harm to children living in their household. Qualitative research has identified a preference among smoking parents whose children are undergoing surgery for a harm-reduction message over cessation. ${ }^{16}$ However, we also know that parents who are able to make their home smoke free, they are subsequently more likely to go on to make a quit attempt. ${ }^{17}$

Families who smoke are potentially reachable within the hospital setting. ${ }^{18}$ There is an expectation among parents in paediatric hospital settings that the physicians will enquire about their smoking, and report that it is acceptable to do this. ${ }^{16}{ }^{19}$ Parents who access care for their child in paediatric emergency departments (EDs) are reported to be motivated to quit and receptive to smoking cessation advice. ${ }^{20-23}$ However, less is known about whether parents, if they are not willing or able to quit smoking, are receptive to SHS harm reduction advice in this setting, although there is qualitative evidence to suggest that the health of the child is as a motivating factor among parents to change their smoking behaviours. ${ }^{24}$ Such hospital contact may, therefore, provide a teachable moment to raise parental awareness of the harms of childhood SHSe and an opportunity to motivate parents to make changes to their smoking behaviours for the benefit of their child's health. $^{18}$

For hospitals to respond to such a teachable moment requires healthcare professionals (HCPs) to play an active role in promoting behavioural change. ${ }^{25}$ Key concepts of a teachable moment include perception of risk, emotional response and self-concept to precipitate change. ${ }^{26} \mathrm{~A}$ mixed-methods systematic review ${ }^{27}$ of interventions to reduce children's exposure to SHS initiated and/or delivered in paediatric secondary care identified five qualitative studies exploring stakeholder experiences allowing identification of barriers and enablers among parents and HCPs. No qualitative research into stakeholders within a UK NHS setting was found.

\section{PRECEDENT STUDY}

\section{Research questions and objectives}

The overall aim of this study, informed by the Capability, Opportunity and Motivation Behaviour (COM-B) mode $^{25}$ is to explore stakeholder views and behavioural change opportunities for a hospital initiated and/or based interventions around supporting parents to change their home smoking behaviours to reduce children's exposure to SHS. This overarching aim will be addressed via two work packages (WP). The aims and objectives for each WP and how they align with COM-B are presented in figure 1 .

\section{METHODS AND ANALYSIS}

\section{Theoretical orientation and study design}

An exploratory qualitative approach, ${ }^{26}$ informed by the COM-B model of behavioural change, ${ }^{25}$ will be used to understand how the paediatric hospital setting can be used to initiate and/or deliver interventions to support parents to change their home smoking behaviours. Due to the lack of existing research in this area and the need for the results to be reflective of the views of stakeholders who could potentially be involved in any consequent interventions a qualitative method is considered appropriate. ${ }^{27}$ As this study is an exploration of initiating a health behavioural change regarding the issue of childhood SHSe in the home environment, a behavioural change informed theory is appropriate to underpin the study development and conduct. The COM-B Model of behavioural change developed by Michie $e t a l^{25}$ as part of a wider behavioural change system known as the 'Behaviour Change Wheel' has been identified for this purpose. The synthesis of existing behavioural change theories led to the identification of three determinants of behaviour $(\mathrm{B})$ change; capability $(\mathrm{C})$, opportunity $(\mathrm{O})$, and motivation $(\mathrm{M}){ }^{25}$

The study has been designed to collect and interpret the data with a qualitative approach in order to understand whether the paediatric hospital visit presents an opportunity for HCPs to deliver a behavioural change intervention and for parents to receive one. That is, does this context present stakeholders with an opportunity to facilitate successful behavioural change; are parents motivated to change their behaviour; and are HCPs are motivated to deliver such an intervention in this setting? Exploring capability should allow for the identification of factors which enable or hinder both parent and HCP stakeholders which can be used to inform the development of interventions/support packages. All participants will also be asked to complete a background questionnaire to provide demographic information, see online supplemental files background HCP and background parents for the specific data collected.

Four researchers are involved in the conduct of this research all of whom have different world views and so will view the data, analysis and interpretation through different lenses. One is a health psychology researcher, 


\begin{tabular}{|l|l|}
\hline Work Package 1a Parents & Work Package 1b Healthcare professionals \\
\hline $\begin{array}{l}\text { Aim: Using the COM-B model to explore } \\
\text { parent/carer knowledge, attitudes, perceptions } \\
\text { and acceptability of a hospital-based intervention } \\
\text { to change their home smoking behaviours. }\end{array}$ & $\begin{array}{l}\text { Aim: Using the COM-B model to explore } \\
\text { healthcare professionals' knowledge, attitudes, } \\
\text { perceptions and acceptability of a hospital-based } \\
\text { intervention to support parents to change their } \\
\text { home smoking behaviours. }\end{array}$ \\
\hline Objectives: & Objectives: \\
\hline $\begin{array}{l}\text { To explore parental knowledge of SHS and } \\
\text { experience of hospital contact around smoking } \\
\text { behaviour. }\end{array}$ & $\begin{array}{l}\text { To explore HCPs knowledge of intervening around } \\
\text { parent home smoking behaviours. }\end{array}$ \\
\hline $\begin{array}{l}\text { To identify parental attitudes and perceptions } \\
\text { towards receiving a hospital-based intervention to } \\
\text { support them to change their home smoking } \\
\text { behaviours. }\end{array}$ & $\begin{array}{l}\text { To identify HCP attitudes and perceptions towards } \\
\text { parent hospital-based intervention to change } \\
\text { their home smoking behaviours. }\end{array}$ \\
\hline $\begin{array}{l}\text { To explore the perceived acceptability among } \\
\text { parents of receiving a hospital-based intervention } \\
\text { to support them to change their home smoking } \\
\text { behaviours. }\end{array}$ & $\begin{array}{l}\text { To explore the perceived acceptability among } \\
\text { HCPs for a parent hospital-based intervention to } \\
\text { change their home smoking behaviours. }\end{array}$ \\
\hline
\end{tabular}

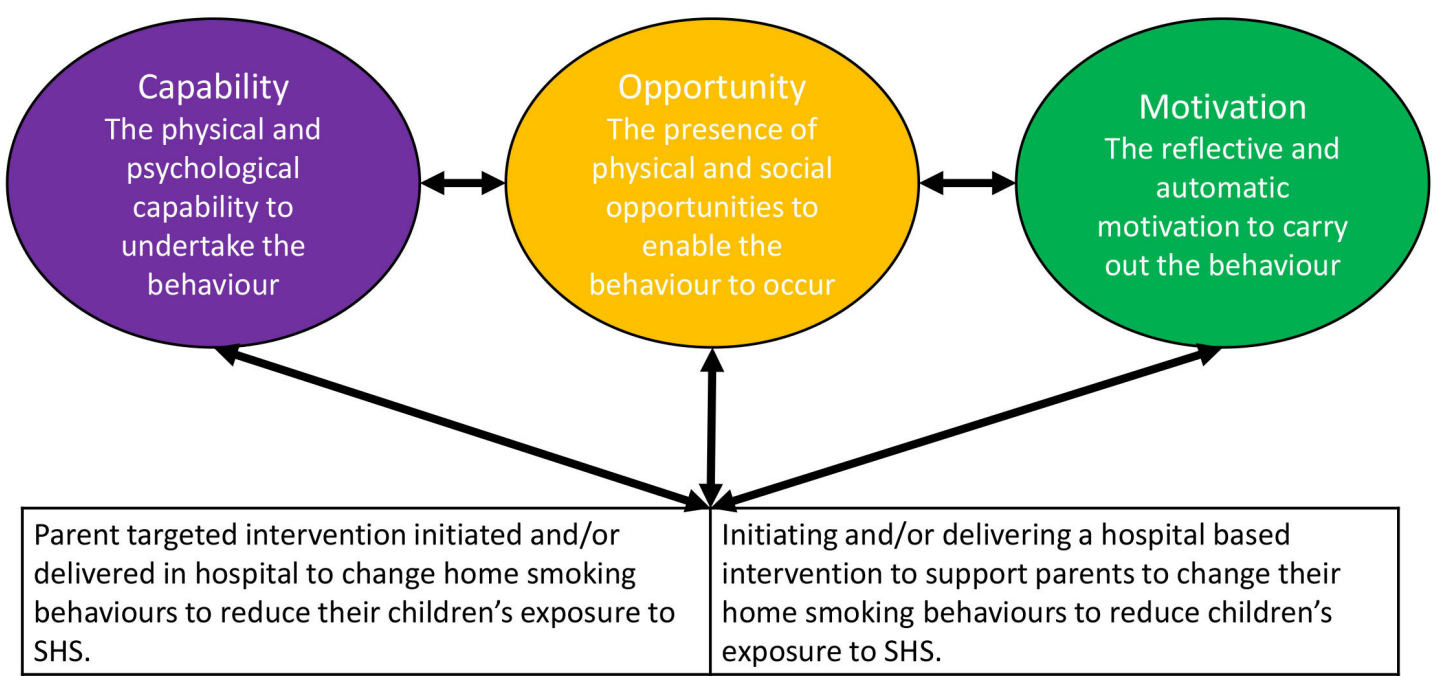

Figure 1 A diagram to illustrate the theoretical underpinning of COM-B to the design of research objectives. COM-B, Capability, Opportunity and Motivation Behaviour; HCPs, healthcare professionals; SHS, secondhand smoke.

one a researcher with expertise in both quantitative and qualitative methods, a public health clinician and a qualitative tobacco control expert. Good reflexive practices will be used throughout including a reflexive diary and memos in NVivo, written and verbal debriefs as and when required. The lead researcher will be independent of the participants.

\section{Study setting and timing}

The study will be undertaken with parents accessing care for their child and HCPs who work at a large Midlands children's hospital (table 1). HCPs and parents will be recruited from any department, with particular emphasis on ED, surgery and ear, nose and throat. The hospital provides secondary, tertiary and national paediatric services. Data collection initially commenced in March 2020; however, it was paused due to the COVID-19 pandemic after data collection from one participant. It is anticipated that the study will resume in spring 2021, with recruitment running throughout summer and autumn 2021, with final analysis and write-up in early 2022.

\section{Eligibility}

\section{Anticipated sample sizes}

Maximum variation purposive sampling twill help to ensure participant diversity and allow a richer exploration of the topic. ${ }^{28}$ For WP1a, participants will be purposively recruited according to their sex, age of their children and which department they present to within the hospital. For WP1b, participants will be purposively recruited according to their sex, professional group and whether they work in planned or unplanned care settings. The number of interviews, will be flexible within each 


\begin{tabular}{|c|c|c|}
\hline Work package & Eligibility & Criteria \\
\hline \multirow[t]{2}{*}{$1 a$} & Inclusion & $\begin{array}{l}\text { Male OR Female AND aged } 18 \text { years and over AND parents/caregiver/guardian AND currently/have } \\
\text { smoked in the home in the last } 5 \text { years AND whose child has presented at Birmingham Children's } \\
\text { Hospital }(\mathrm{BCH}) \text { within the last } 5 \text { years AND can speak English AND has the capacity to provide informed } \\
\text { consent }\end{array}$ \\
\hline & Exclusion & $\begin{array}{l}\text { Parents who only use smokeless tobacco or electronic cigarettes at home OR parents who have had a } \\
\text { completely smoke-free home for the past } 5 \text { years or longer }\end{array}$ \\
\hline $1 b$ & Exclusion & - \\
\hline
\end{tabular}

group (parents and HCPs) and the adequacy of the final samples carefully monitored during the research process to ensure that the overall sample and associated data had sufficient information power to develop new knowledge in relation to the research questions. ${ }^{29}$ The size of the sample will ultimately be determined by the number of interviews/discussion groups required in order to ensure that we have sufficiently rich data to answer the research questions. ${ }^{30}$ However, an estimate of $15-20$ parents and 20-25 HCPs is anticipated to be sufficient.

\section{Sampling and recruitment}

\section{Work package 1a}

Within WP1a, we will aim to sample parents who present for both planned hospital visits (eg, surgery) and unplanned hospital visits (eg, ED). There will ideally be a mixture of parents with children aged 5 years and under (who are typically the most exposed and particularly within the home environment $)^{5}$ and children aged 6-17 years.

Posters advertising the study will be displayed across identified departments within the recruiting NHS Trust. Social media will be used for example via Facebook and Twitter as well as the Trust Website to advertise the study. Potential parent participants who present in the hospital will be approached in the first instance by a member of their child's usual care team. Potential participants will be asked to sign a written agreement for their contact details to be recorded, securely stored and used to contact them to arrange an interview/discussion group. Self-identifying participants, for example, those responding to the study advert will be asked for verbal consent to the storage and use of their contact details as part of this study. Brief participant information leaflets (PILs) will be made available at reception desks and in communal waiting areas, they may also be sent with appointment letters for targeted clinics such as respiratory. Additionally, snowball sampling will be used with eligible participants asked to pass on the study details to their friends or family who they feel may be eligible and interested in participating in this study. ${ }^{31}$

\section{Work package $1 \mathrm{~b}$}

Within WP1b, we will aim to have HCPs who work in inpatient hospital settings and those who work in outpatient hospital settings. There will ideally be a range of HCPs included (eg, doctors, nurses and allied health professionals).

HCPs at the recruiting NHS Trust will be contacted directly about the study through personal and professional networks of the research team members. This study will also be advertised via posters in the hospital, on social media for example via Facebook and Twitter, the Trust intranet and in email newsletters. In the advert, potential participants will be asked to contact the research team directly via phone or email. Participants will be invited to disseminate the study information (snowballing) ${ }^{32}$ to any colleagues who may be eligible and interested in taking part.

Following expressions of interest, potentially eligible participants from both WP1a and WP1b will be contacted by a member of the study team via email, phone, SMS and/or post to confirm eligibility, establish their preferred method (interview or discussion group) and format (videoconferencing such as Zoom or telephone) and to arrange a mutually convenient time and location for data collection.

\section{Informed consent}

All participants will be asked to give written, electronic and/or verbally recorded (for those completing interviews on the phone/via videoconferencing platforms) informed consent to participate in the study at the beginning of the interview/discussion group. The agreement to consent will follow the provision of a written explanation of the study (PIL) and an opportunity to ask a member of the research team questions about the study either faceto-face, via videoconferencing, via the telephone or via email communication. For videoconferencing and telephone interviews, a consent form will be posted (with a return envelope) or emailed to the participants who can sign and return electronically indicating their consent to participate (this electronic consent may include an electronic signature rather than wet ink signature). If the written consent form cannot be obtained (eg, for logistical reasons) then the consent form will be read out to the participant verbally at the start of the interview and their responses audio recorded and documented. It will 
be made clear that involvement in the study is voluntary and that they are free to withdraw from the study without giving a reason. We will however keep any information already obtained and use these data anonymously.

\section{Data collection}

Participants will be asked about their preferred method for participating in the study either via an interview or a discussion group which will be audio recorded. Data collection will be facilitated via the use of guides developed from the literature and informed by the COM-B model of behavioural change. ${ }^{25}$ Interviews and discussion groups will be conducted in a participant-focused manner allowing experiences and views important to participants to emerge naturally. ${ }^{33}$ Table 2 (parents) and table 3 (HCPs) provide a summary of the areas that will be explored in interviews/discussion groups. Interviews are

Table 2 Provisional discussion guide for parent interviews/discussion groups

\section{Objective}

1a(a) To explore parent knowledge of SHS and experience of hospital contact.

\section{Capability (physical and psychological)}

How have you used the hospital in the past with your child?

- Did the doctors/nurses talk to you about smoking?

Explore what they know about SHS

-What if anything would you like to know?

Do you feel that HCPs would be able to help?

Why (not)?

- Does it vary between roles and seniority?

Opportunity (physical and social)

Do you perceive addressing SHSe as part of your job?

- Yes/no

- Why

Motivation (reflective and automatic)

Do you feel that it is important to reduce children's exposure to SHS?

Explore current home smoking behaviours

- Any countermeasures

- Any barriers and facilitators they have come across

1a(b) To identify parent attitudes and perceptions towards receiving a hospital-based intervention to support them to reduce smoking in the home.

Capability (physical and psychological)

To be explored

Opportunity (physical and social)
Where would you like a support package to be delivered?

- Eg, at home, in the hospital

- Why

When would you feel it is appropriate for a support package to be delivered?

- Why

How would you like a support package to be delivered?

- Face to face, over the phone

- Why

What would you like a support package to include?

- Eg, bio feedback, information

Motivation (reflective and automatic)

Would there be any negative impact of receiving an intervention in this setting?

Do you want to receive support for SHS from a HCP?

\begin{tabular}{|c|c|}
\hline \multirow{6}{*}{$\begin{array}{l}\text { 1a(c) To explore the perceived } \\
\text { acceptability among parents of } \\
\text { receiving a hospital-based intervention } \\
\text { to reduce smoking in the home. }\end{array}$} & Capability (physical and psychological) \\
\hline & To be explored \\
\hline & Opportunity (physical and social) \\
\hline & $\begin{array}{l}\text { Would it be acceptable to be approached in the hospital about a support package for SHS? } \\
\text { Why? }\end{array}$ \\
\hline & Motivation (reflective and automatic) \\
\hline & $\begin{array}{l}\text { Who do you think should support parents to reduce SHS in the home? } \\
\text { HCPs? } \\
\text { Families? }\end{array}$ \\
\hline Intervention development & $\begin{array}{l}\text { What support parents would be happy to receive (or not) in this setting } \\
\text { Why (not)? }\end{array}$ \\
\hline
\end{tabular}

HCPs, healthcare professionals; SHS, secondhand smoke. 
Table 3 Provisional discussion guide for HCP interviews/discussion groups

\section{Objective}

Current practice

$1 \mathrm{~b}(\mathrm{a})$ To explore

HCPs knowledge and skills of intervening around children's exposure to SHS in the home.
Explore their practices around SHS exposure

Do you see parents who smoke in the home?

- How do you identify these families?

-What do you normally do if you identify these families?

- Is it usual practice currently to ask and/or address parental smoking of patients? Capability (physical and psychological)

Explore their knowledge about SHS and SHS exposure in children

- Could you tell me what you know about SHS in the home?

- Do you feel you are knowledgeable about SHS?

Why (not)?

Do you feel you have the necessary skills to intervene around SHS?

Why (not)?

Are there any skills/education that would help them to intervene around SHS?

Opportunity (physical and social)

To be explored

Motivation (reflective and automatic)

To be explored Capability (physical and psychological)

To be explored

Opportunity (physical and social)

Explore any factors that might make it difficult to deliver a SHS intervention

- Eg, work environment, colleagues

Explore any factors that might make it easier to deliver a SHS intervention

- Eg, work environment, colleagues

Motivation (reflective and automatic)

How would you feel about delivering a SHS intervention to parents?

Why (not)?

What do you think an intervention should include?

- Eg, education, motivational interviewing
$1 \mathrm{~b}(\mathrm{c})$ To explore the perceived acceptability among HCPs for a parent's hospital-based intervention to reduce childhood exposure to SHS in the home.

Capability (physical and psychological)

To be explored

Opportunity (physical and social)

Do you think parents would be receptive to an intervention?

Do you think other HCPs would be willing to intervene?

Motivation (reflective and automatic)

Who do you think should support parents to reduce SHS?

- HCPs? Vary across department/role?

- Parents responsibility?

Intervention development

Why (not)?

HCPs, healthcare professionals; SHS, secondhand smoke.

expected to last up to $60 \mathrm{~min}$ and discussion groups up to $90 \mathrm{~min}$. The discussion guide will be refined iteratively to facilitate exploration of evolving ideas, this process may be repeated throughout the course of data collection. Data collection and analysis will take place concurrently ${ }^{26}$ and will continue until the research team judge that the data and sample have sufficient depth and breadth to address the research objectives. ${ }^{30}$

Interviews and discussion groups be will run virtually via an appropriate platform such as Zoom/Teams or via phone (interviews only). If possible and within COVID-19 guidance, we may run interviews and discussion groups face to face in appropriate prespecified locations, for example, in a private room at the hospital where they were recruited, or in a private room at the University where the researchers are based or in the participants own home (interviews only).

\section{Inconvenience allowances and expenses}

Work package 1a

Interview/discussion group participants will be reimbursed for reasonable travel expenses to and from the 
interview/discussion group venue (only where the venue is away from their home address). Discussion group participants will also be provided with refreshments. All participants will be given a £20 high street voucher at the end of the interview/discussion group to reimburse them for their time.

\section{Work package $1 \mathrm{~b}$}

HCPs will be reimbursed for reasonable travel expenses should data collection occur away from their place of work. Discussion group participants will also be provided with refreshments.

\section{Data analysis}

Study data collected from interviews and discussion groups will be examined and interpreted via the framework method established by Ritchie $e t a \ell^{34}$ and further described by Gale et $a l^{35}$ The framework method has been advocated for use in multidisciplinary health research and provides a systematic and flexible (to theoretical positions) model for managing and mapping qualitative data from multiple sources allowing for both inductive and deductive analyses. ${ }^{35} 36$ Audiorecordings will be transcribed and anonymised. Recordings will be transcribed either by a member of the research team, or an external transcription company who will sign a data storage and confidentiality agreement. Transcriptions will then be imported into NVivo software to facilitate data management. ${ }^{37}$

Following familiarisation with the transcripts line-byline deductive coding against the COM-B framework while also allowing for an 'other' and an 'intervention' code. This will allow for data that does not fit within COM-B as well as data on what potential interventions may look like. Analyst triangulation ${ }^{38}$ will be achieved via a random selection of the deductively coded transcripts sent to co-authors for coding review and subsequent discussion. This will be followed by a line-by-line inductive analysis within COM-B plus the 'other' and 'intervention' code across all transcripts. Once all transcripts have been coded within the COM-B, other and intervention deductive framework, further inductive coding will be undertaken within each of these domains to identify themes and sub-themes. A random selection of these inductively coded transcripts will be sent to co-authors for coding review and subsequent discussion.

Once the final code book has been generated and agreed it will be applied to all transcripts and a framework matrix will then be constructed. This matrix is likely to have each case (grouped by stakeholder) as a row and subthemes, themes and COM-B, other and intervention domains as the column headers. The generation of a matrix will provide an intuitively structured overview of the data and facilitate exploration of patterns within and between data. As it is not aligned with an epistemological, philosophical or theoretical approach the Framework Approach is compatible with the COM-B model of behavioural change. $^{25}$

\section{Patient and public involvement}

During the design and development of the study protocol, parents from the recruiting Trust's family advisory group were sent all patient facing documents to feedback on, and supported the choice of a preferred logo and design. We will explore opportunities for further patient and public involvement.

\section{ETHICS}

\section{Risk assessment and management}

The University of Birmingham is the nominated sponsor and data controller for the study. There is a possibility that topics of a sensitive or challenging nature could arise in the discourse, although this is not anticipated. In these circumstances, participants will be offered a short break or a change of subject. At all times, the welfare of the participants will be placed ahead of the knowledge to be gained.

Parents will be explicitly informed that their involvement in the study will in no way influence their child's care. All participants will be made aware via the PIL that should there be any disclosure of concern that the interviewer/facilitators will discuss this with the wider research team who will act accordingly.

\section{Data security}

All study researchers will uphold the core principles and comply with the requirements of the Data Protection Act 2018 and the European Union General Data Protection Regulation 2016/679 in the collection, storage, processing and disclosure of personal information. Researchers will also maintain up to date Good Clinical Practice training.

Study data will only be accessible to members of the research team and relevant regulatory bodies. Personal identifiable data, including audio recordings will be safely destroyed when the main results of the study are published. It is anticipated that this will be within approximately 12 months of the end of the study, but may take longer. Anonymised study data will be retained for ten years following the publication of the final study report in accordance with University of Birmingham policy.

\section{DISSEMINATION}

The data collected as part of this study will be owned by the sponsor, the University of Birmingham. A final study report will be prepared for publication in targeted journals, as well as presented at relevant conferences and events. The final study report will also feed into a doctoral thesis, which will be publicly accessible. Results of this research may be used to directly inform future funding applications and proposals.

Twitter Laura Jones @drlauraljones

Contributors All authors were involved in the design of the study. EF wrote the study protocol, LJ, CCh and CCu provided feedback and approved the final version. 
Funding This study is funded as part of a PhD studentship by the Birmingham Children's Hospital Charity (BCHRF519).

Disclaimer The funders will take no role in study design, data collection and analysis, decision to publish, or preparation of publications.

Competing interests None declared.

Patient consent for publication Not applicable.

Ethics approval The study has received a favourable opinion from the East Midlands Leicester Central Research Ethics Committee (19/EM/0171) and approval from the Health Research Authority. Voluntary informed consent to participate and be audio-recorded will be gained from all participants.

Provenance and peer review Not commissioned; externally peer reviewed.

Supplemental material This content has been supplied by the author(s). It has not been vetted by BMJ Publishing Group Limited (BMJ) and may not have been peer-reviewed. Any opinions or recommendations discussed are solely those of the author(s) and are not endorsed by BMJ. BMJ disclaims all liability and responsibility arising from any reliance placed on the content. Where the content includes any translated material, BMJ does not warrant the accuracy and reliability of the translations (including but not limited to local regulations, clinical guidelines, terminology, drug names and drug dosages), and is not responsible for any error and/or omissions arising from translation and adaptation or otherwise.

Open access This is an open access article distributed in accordance with the Creative Commons Attribution Non Commercial (CC BY-NC 4.0) license, which permits others to distribute, remix, adapt, build upon this work non-commercially, and license their derivative works on different terms, provided the original work is properly cited, appropriate credit is given, any changes made indicated, and the use is non-commercial. See: http://creativecommons.org/licenses/by-nc/4.0/.

\section{ORCID iDs}

Erica Ferris http://orcid.org/0000-0001-5666-0460

Laura Jones http://orcid.org/0000-0002-4018-3855

\section{REFERENCES}

1 Öberg M, Jaakkola MS, Woodward A, et al. Worldwide burden of disease from exposure to second-hand smoke: a retrospective analysis of data from 192 countries. Lancet 2011;377:139-46.

2 US Department of Human and Health Services. The health consequences of involuntary exposure to tobacco smoke: a report of the surgeon General. Atlanta, GA: US Department of Health and Human Services, Centers for Disease Control and Prevention, Coordinating Center for Health Promotion, National Center for Chronic Disease Prevention and Health Promotion, Office on Smoking and Health, 2006.

3 WHO. Tobacco key facts, 2019. Available: https://www.who.int/ news-room/fact-sheets/detail/tobacco [Accessed 6 Oct 2019].

4 Sims M, Tomkins S, Judge K, et al. Trends in and predictors of second-hand smoke exposure indexed by cotinine in children in England from 1996 to 2006. Addiction 2010;105:543-53.

5 Jarvis MJ, Feyerabend C. Recent trends in children's exposure to second-hand smoke in England: cotinine evidence from the Health Survey for England. Addiction 2015;110:1484-92.

6 Jones LL, Hashim A, McKeever T, et al. Parental and household smoking and the increased risk of bronchitis, bronchiolitis and other lower respiratory infections in infancy: systematic review and metaanalysis. Respir Res 2011;12:5.

7 Jones LL, Hassanien A, Cook DG, et al. Parental smoking and the risk of middle ear disease in children: a systematic review and metaanalysis. Arch Pediatr Adolesc Med 2012;166:18-27.

8 Murray RL, Britton J, Leonardi-Bee J. Second hand smoke exposure and the risk of invasive meningococcal disease in children: systematic review and meta-analysis. BMC Public Health 2012;12:1062.

9 Chiswell C, Akram Y. Impact of environmental tobacco smoke exposure on anaesthetic and surgical outcomes in children: a systematic review and meta-analysis. Arch Dis Child 2017;102:123-30.

10 International Agency for Research on Cancer. IARC monographs on the evaluation of carcinogenic risks to humans, in IARC. Lyon, 2004: 1-1452.
11 Vineis P, Airoldi L, Veglia F, et al. Environmental tobacco smoke and risk of respiratory cancer and chronic obstructive pulmonary disease in former smokers and never smokers in the EPIC prospective study. BMJ 2005;330:277.

12 Leonardi-Bee J, Jere ML, Britton J. Exposure to parental and sibling smoking and the risk of smoking uptake in childhood and adolescence: a systematic review and meta-analysis. Thorax 2011;66:847-55.

13 Royal College of Physicians. Passive smoking and children. A report by the tobacco Advisory Grou. London: RCP, 2010.

14 Jarvis MJ, Mindell J, Gilmore A, et al. Smoke-free homes in England: prevalence, trends and validation by cotinine in children. Tob Control 2009;18:491-5

15 Jones LL, Atkinson O, Longman J, et al. The motivators and barriers to a smoke-free home among disadvantaged caregivers: identifying the positive levers for change. Nicotine \& Tobacco Research 2011;13:479-86.

16 Warner DO, Campbell EB, Hathaway JC, et al. Reducing secondhand smoke exposure of children undergoing surgery. Am J Health Behav 2014;38:924-32.

17 Ratschen E, Thorley $\mathrm{R}$, Jones $\mathrm{L}$, et al. A randomised controlled trial of a complex intervention to reduce children's exposure to secondhand smoke in the home. Tob Control 2018;27:155-62.

18 Winickoff JP, Berkowitz AB, Brooks K, et al. State-of-the-art interventions for office-based parental tobacco control. Pediatrics 2005;115:750-60.

19 Abdullah AS, Ma Z, Liao J, et al. Addressing parental smoking in pediatric settings of Chinese hospitals: a qualitative study of parents. Biomed Res Int 2014;2014:1-8.

20 Kanis J, Byczkowski T, Mahabee-Gittens EM. Motivation to quit smoking in parental smokers in the pediatric emergency department. Pediatr Emerg Care 2014;30:546-51.

21 Mahabee-Gittens EM, Gordon J. Acceptability of tobacco cessation interventions in the pediatric emergency department. Pediatr Emerg Care 2008;24:214-6.

22 Mahabee-Gittens EM, Stone L, Gordon JS. Pediatric emergency department is a promising venue for adult tobacco cessation interventions. Nicotine Tob Res 2013;15:1792-3.

23 Mahabee-Gittens M. Smoking in parents of children with asthma and bronchiolitis in a pediatric emergency department. Pediatr Emerg Care 2002;18:4-7.

24 Lee MT, Bracamontes J, Mosier E, et al. Preferred smoking cessation methods for Asian or Pacific Islander household smokers who live with hospitalized children. Hawaii Med J 2011;70:48.

25 Michie S, van Stralen MM, West R. The behaviour change wheel: a new method for characterising and designing behaviour change interventions. Implementation Sci 2011;6:42.

26 DiCicco-Bloom B, Crabtree BF. The qualitative research interview. Med Educ 2006;40:314-21.

27 Creswell JW, Hanson WE, Clark Plano VL, et al. Qualitative research designs. Couns Psychol 2007;35:236-64.

28 Patton MQ. Qualitative research \& evaluation methods: Integrating theory and practice. Sage Publications, 2014.

29 Malterud K, Siersma VD, Guassora AD. Sample size in qualitative interview studies: guided by information power. Qual Health Res 2016;26:1753-60.

30 Malterud K, Siersma VD, Guassora AD. Sample size in qualitative interview studies. Qual Health Res 2016;26:1753-60.

31 Etikan I, Alkassim R, Abubakar S. Comparision of snowball sampling and sequential sampling technique. Biom Biostat Int $J$ 2016;3:55.

32 Rubin A, Babbie ER. Empowerment series: research methods for social work. Cengage Learning, 2016.

33 Braun V, Clarke V. Successful qualitative research: a practical guide for beginners. Sage, 2013.

34 Ritchie J, Lewis J, Nicholls CMN, et al. Qualitative research practice: a guide for social science students and researchers. Sage, 2013.

35 Gale NK, Heath G, Cameron E, et al. Using the framework method for the analysis of qualitative data in multi-disciplinary health research. BMC Med Res Methodol 2013;13:117.

36 Ritchie J, Spencer L. Qualitative data analysis for applied policy research, in analyzing qualitative data. Routledge, 2002: 187-208.

37 QSR International, P.L. NVivo qualitative data analysis software Version 122020.

38 Carter N, Bryant-Lukosius D, DiCenso A, et al. The use of triangulation in qualitative research. Oncol Nurs Forum 2014;41:545-7. 